\title{
Determining Size Distributions and Composition of Particles Suspended in Water: A New SEM-EDS Protocol with Validation and Comparison to Other Methods
}

\author{
Heather Groundwater And Michael S. Twardowski \\ Department of Research, WET Labs, Inc., Narragansett, Rhode Island \\ HEIDI M. DIERSSEN \\ Department of Marine Science, University of Connecticut, Groton, Connecticut \\ ANTOINE SCIANDRA \\ Laboratoire d'Océanographie de Villefranche, Villefranche-sur-Mer, France \\ SCOTT A. FREEMAN \\ Department of Research, WET Labs, Inc., Narragansett, Rhode Island
}

(Manuscript received 16 February 2011, in final form 26 October 2011)

\begin{abstract}
Knowledge of particle size distributions (PSDs) in seawater is important for understanding several facets of marine science, such as the behavior of light scattering in seawater, phytoplankton dynamics, and biogeochemical cycling. Here, a method has been developed to quantify the size distribution of particle suspensions and characterize their chemical composition utilizing a scanning electron microscope (SEM) coupled with an energy dispersive spectrometer (EDS) and applying image analysis techniques, including automatic thresholding. The method was validated by verifying the PSD and chemical composition of the Arizona Test Dust (ATD), which has a well-documented size distribution and chemical composition. Size distributions of ATD particles containing specific elements important in the marine environment, such as silicon, iron, calcium, aluminum, and potassium, were quantified. PSDs determined with the technique in field samples from coastal Long Island Sound and the remote South Pacific were compared with other sizing methods, including electroresistivity and laser diffractometry. Most accurate results for PSD determinations occurred when the particle mass loading on the filter was between 0.04 and $0.1 \mathrm{mg} \mathrm{cm}^{-2}$. With this in mind, immediate feedback in the field can be provided to prepare appropriate filtration sample volumes due to a linear relationship between the beam attenuation coefficient at $650 \mathrm{~nm}\left(c_{650}\right)$ and the total suspended matter (TSM). Overall, the method presents two defining advantages in 1) minimizing user bias, because the majority of the analysis is automated, and 2) providing an elemental distribution in the context of a particle size distribution.
\end{abstract}

\section{Introduction}

Assessing particle size distributions (PSDs) in seawater is relevant to understanding how light scatters in the ocean (Jonasz and Fournier 2007). Scattering by particles affects light propagation through the ocean via radiative transfer (Mobley et al. 1993; Zaneveld et al. 2005), exerts primary control of the bidirectional reflectance distribution function

Corresponding author address: Heather Groundwater, 70 Dean Knauss Drive, WET Labs, Inc., Narragansett, RI 02882.

E-mail: heather@wetlabs.com
(BRDF) that contributes to remotely sensed reflectance by satellite imagers (Morel and Gentili 1993; Morel et al. 1995), and allows quantification of particle characteristics, such as concentration and the bulk refractive index (similar to particle density), through inversion (Twardowski et al. 2001, 2005, 2007, 2012; Zhang et al. 2011). Recently, ocean color remote sensing images were used to retrieve PSDs over regional and global scales (Kostadinov et al. 2009). Knowledge of PSDs also aids in understanding phytoplankton ecology and biogeochemical cycling (Alldredge and Gotschalk 1988; Chisholm 1992; Wells and Goldberg 1992, 1994; Jackson et al. 1997; Pilskaln et al. 1998). 
Particle size distributions of aquatic samples have been determined utilizing several different methods (Syvitski 1991; Allen 1997; Reynolds et al. 2010), including microscopy (optical and electron), electrical resistance sensing, optical diffractometry, flow cytometry, and field-flow fractionation. For each method there is an assortment of commercially available instruments that can be used, each with their own specific advantages and disadvantages. Methods other than microscopy typically do not measure the actual size and shape of the particles, but volume displaced or an impinged cross-sectional area, which are then usually explicitly or implicitly converted to an equivalent spherical diameter (ESD). These methods also typically cannot adequately resolve the submicron particle population, with the exception of field-flow fractionation.

Particle size determination by image analysis has often been considered the definitive method because individual particles are analyzed directly (Allen 1997; Santschi et al. 1998; Wells and Goldberg 1992; Doucet et al. 2005). There are various methods of microscopy used today, and they all generally share similar disadvantages and advantages. Traditionally, preparing a sample for microscopic analysis can be labor intensive. For instance, a sample prepared for scanning electron microscopy (SEM) image analysis may be stained with a heavy metal so as to produce a better binary image and provide sample preservation during SEM analysis (Lavoie 1992). A method of sample preparation for transmission electron microscopy (TEM) requires up to $4 \mathrm{~h}$ of centrifugation time for adequate sedimentation of particles sized between $5 \mathrm{~nm}$ and $40 \mu \mathrm{m}$ (Wells and Goldberg 1992). Samples prepared for atomic force microscopy (AFM) require adequate particle adsorption to the surface substrate so as to minimize the movement of particles (Doucet et al. 2004; Santschi et al. 1998; Wilkinson et al. 1999).

In addition to extensive sample preparation, quantification of particle sizes by means of microscopy may have inherent errors resulting from the nature of image analysis. The quality of data relies heavily on the quality of particle dispersion on a substrate such that there cannot be too many particles, otherwise there will be issues with overlapping (Allen 1997). As a particle is deposited on a substrate it will tend to lie flat on the side with the largest area cross section and, in doing so, the nonsphericity of a particle generally causes the projected particle sizes to be larger than an equivalent randomly oriented "mean" size (Jonasz 1987). Similarly, overestimates of particle size may occur from AFM measurements resulting from the shape of the instrument probe (Doucet et al. 2004; Santschi et al. 1998; Wilkinson et al. 1999). Enough particles must also be resolved across the entire population to minimize population statistical uncertainties (Sournia 1978; Allen 1997). The most notable advantage in assessing samples with microscopy is the fact that an actual image of the particles is captured. After an image is acquired, particle sizes are usually determined manually or automatically with the aid of an image analysis program (Allen 1997). Typically, a threshold is applied to images to segment particles from the background (Sezgin and Sankur 2004). SEM and environmental scanning electron microscopy (ESEM) qualitatively demonstrated that water is a significant component of the structure of aquatic colloids and particles (Doucet et al. 2005). ESEM is essentially an SEM that operates at low pressures so that water remains in a liquid phase (Danilatos 1988). Doucet et al. (2005) suggested that capturing images of hydrated samples through ESEM produced more realistic PSDs than images from SEM analysis because particles were not dehydrated.

Information on the composition of individual particles can also be obtained both visually and chemically if an energy dispersive X-ray spectrometer [energy dispersive spectrometer (EDS, EDX, EDXS)] is used in conjunction with an SEM (Lambert et al. 1981; Bishop and Biscaye 1982; De Boer and Crosby 1995; Kahn et al. 2002; Kutchko and Kim 2006). EDS operates by exciting ground-state electrons, and the energy released as X-rays corresponds to particular elements. Typically, a bulk analysis of elements can be determined by looking at the counts per element in relation to total counts, or an image can be produced that highlights or maps pixels locating a particular element. Recently, a method has been developed to determine particle sizes immediately during image acquisition, not from a saved image, using scanning electron microscopy with automated image analysis and X-ray energy spectroscopy (SAX; see Lavoie 1992; Peng et al. 2002; Peng et al. 2007). While the sample is loaded in the SEM, the particle analysis software controls both the image analysis and the X-ray microanalysis. Filters are treated beforehand with a chemical coating to produce a better-contrasted binary image for the automatic program to detect particles from the background and also to avoid charging and heat damage to the particles. An earlier study of estuarine particles utilized a similar set up with an SEM linked with an electron probe X-ray microanalysis (EPXMA; see Bernard et al. 1986). Estuarine particles were characterized via an automated multivariate cluster analysis that placed particles into geochemically relevant groups.

Existing SEM-EDS methods must be refined for determining oceanic PSDs. Optimized particle loading on a filter is key to obtaining good images for analysis, yet there currently is no way of quantifiably gauging loading in the field. Validation and comparison of particle sizes to standard materials and other sizing methods can be 
important for evaluating errors, yet this has rarely been carried out. Methods typically also involve some subjectivity in setting contrast thresholds by the operator, which could lead to different results for the same sample and the same equipment.

A method is described for determining oceanic particle size distributions and particle compositions utilizing a scanning electron microscope coupled with an energy dispersive spectrometer and a computer image analysis program. Backscattered electrons were generated to capture images so that samples required no special chemical treatment prior to image analysis. The time required to process particle sizes and elemental compositions from the SEM and EDS images was on the order of minutes because they were determined with the use of automated scripts utilizing MATLAB's Image Analysis Toolbox (http://www.mathworks.com/). Results of the method were compared with the PSD and elemental composition of Arizona Test Dust (ATD) provided by the manufacturer. The method was then applied to determining PSDs of field samples collected from the coastal Long Island Sound and from the remote South Pacific. Additionally, these results provided insight into sampling methods that would produce optimal results for SEM and EDS testing.

\section{Methods}

\section{a. Sample collection}

Samples from the field were collected with a Niskin bottle. After retrieval of the bottle, it was held nearly horizontal while the end cap was loosened to gently empty the entire contents into an acid and base prewashed polycarbonate carboy. The carboy was first rinsed with the sample twice. The sample in the carboy was gently swirled before any subsamples were collected. In preparation for imaging and total suspended mass (TSM) analyses, suspended particles from the carboy subsamples were collected by vacuum filtration onto preweighed Poretics polycarbonate $0.4-\mu \mathrm{m}$ filters. Subsample volumes were measured with a graduated cylinder. Subsample filtration occurred immediately upon the sample collection or within a couple of hours of sample collection. Filters were then rinsed with distilled water to remove any dissolved ions from solution and refrigerated in disposable plastic Petri dishes. Before analysis later in the laboratory, the filters were dried for $2 \mathrm{~h}$ in an oven at $50^{\circ} \mathrm{C}$. TSM was obtained with a Fisher Scientific accu-124D dualrange balance with $0.01-\mathrm{mg}$ uncertainty.

\section{b. Sample sources}

For laboratory validation analyses, suspensions of ATD, International Organization for Standardization
(ISO) Standard 12103-1, and A1 Ultrafine Test Dust [Powder Technology, Inc. (PTI)] were prepared by diluting initial slurries of test dust in purified water. The Ultrafine Test Dust product is provided with a factory-measured size distribution (via a Coulter Counter) with an absolute maximum diameter cutoff of $10 \mu \mathrm{m}$. ATD is compositionally diverse and very similar to mineral particles encountered in natural oceanic environments. An analytical blank was also prepared following similar filtration procedures with only the purified water. Field samples were collected from Long Island Sound, New York, in 2005 and from the South Pacific Ocean in 2004 during the Biogeochmistry and Optics South Pacific Experiment (BIOSOPE) cruise leg from Easter Island to Concepcion, Chile (Table 1). Details of the BIOSOPE cruise can be found in a special volume of Biogeochemistry (2008, Vol. 5; see Claustre et al. 2008).

For Long Island Sound, PSDs were also immediately resolved with a traditional electroresistance particle analyzer (Elzone, Micrometrics) and an in situ laser diffraction particle size analyzer (LISST-100X, type B, Sequoia Scientific, Inc.). The Elzone was fitted with either a 30 - or a $120-\mu \mathrm{m}$ orifice, resolving particles in approximately the 1-24.4- and 1.9-45.4- $\mu \mathrm{m}$ size ranges, respectively. The $30-\mu \mathrm{m}$ orifice had a sample rate of $0.17 \mathrm{~mL} \mathrm{~min}^{-1}$ and the $120-\mu \mathrm{m}$ orifice had a sample rate of $3.4 \mathrm{~mL} \mathrm{~min}^{-1}$. A 10-point moving average was applied to Elzone data. For LISST-100X, scattered light in the near-forward angles is measured on concentric detector rings and inversion modeling yields the volumetric particle concentration in 32 logarithmically spaced size classes. The volume distribution represents the equivalent volume sphere representation of the true particle assemblage.

For the South Pacific, PSDs were also immediately analyzed with a Multisizer 3 Coulter Counter (Beckman Coulter, Inc.). The Coulter Counter was fitted with a $30-\mu \mathrm{m}$ orifice resolving particles in approximately the $0.6-18-\mu \mathrm{m}$ size range. Occasionally, it was fitted with a $20-\mu \mathrm{m}$ orifice and resolved particles as small as $0.5 \mu \mathrm{m}$. Results obtained with both aperture tubes for the same sample were consistent within the overlapping size range. The Coulter Counter was set up in a dry laboratory in a way that ship vibrations were efficiently reduced. Background counts were routinely checked by measurements of clear seawater filtered multiple times through a $0.2-\mu \mathrm{m}$ filter. Background counts with filtered seawater were always less than $5 \%$ of the sample counts, with a mean value of $2.7 \%$, and were not used to correct PSD data because most of this signal was likely due to unavoidable remnant particles in the filtrate. The final PSD for a given sample was calculated by 
TABLE 1. Sample collection location and filter data for Long Island Sound and South Pacific samples.

\begin{tabular}{|c|c|c|c|c|c|c|}
\hline Station & Lat $\left({ }^{\circ}\right)$ & Lon $\left(^{\circ}\right)$ & Date & Depth (m) & $\operatorname{TSM}\left(\mathrm{g} \mathrm{m}^{-3}\right)$ & Particle mass (mg) \\
\hline \multicolumn{7}{|c|}{ Long Island Sound } \\
\hline 08 & 41.253 & -72.342 & 17 May 2005 & 1 & 2.96 & 1.48 \\
\hline 07 & 41.253 & -72.342 & 17 May 2005 & 1 & 3.32 & 2.26 \\
\hline 12 & 41.143 & -72.620 & 17 May 2005 & 1 & 3.05 & 1.22 \\
\hline 14 & 41.216 & -72.906 & 17 May 2005 & 1 & 4.23 & 1.69 \\
\hline 20 & 41.054 & -73.331 & 18 May 2005 & 1 & 2.17 & 0.65 \\
\hline 23 & 40.917 & -73.661 & 18 May 2005 & 1 & 4.36 & 0.96 \\
\hline 24 & 40.489 & -74.096 & 19 May 2005 & 1 & 4.59 & 1.29 \\
\hline 26 & 40.498 & -73.895 & 19 May 2005 & 1 & 0.85 & 0.34 \\
\hline 28 & 40.488 & -73.938 & 19 May 2005 & 1 & 2.13 & 0.85 \\
\hline \multicolumn{7}{|c|}{ South Pacific } \\
\hline $42 \mathrm{~A}$ & -9.030 & -136.944 & 1 Nov 2004 & 5 & 0.089 & 0.25 \\
\hline $18 \mathrm{~A}$ & -23.542 & -117.874 & 10 Nov 2004 & 5 & 0.118 & 0.33 \\
\hline $23 \mathrm{~A}$ & -30.034 & -98.393 & 23 Nov 2004 & 5 & 0.046 & 0.13 \\
\hline $12 \mathrm{~A}$ & -30.775 & -95.444 & 24 Nov 2004 & 5 & 0.099 & 0.50 \\
\hline $3 \mathrm{~A}$ & -31.843 & -91.461 & 26 Nov 2005 & 5 & 0.100 & 0.28 \\
\hline $7 \mathrm{~A}$ & -31.895 & -91.366 & 29 Nov 2004 & 5 & 0.155 & 0.31 \\
\hline $5 \mathrm{~A}$ & -32.696 & -84.074 & 2 Dec 2011 & 5 & 0.096 & 0.27 \\
\hline $48 \mathrm{~A}$ & -33.356 & -78.104 & 4 Dec 2004 & 5 & 0.121 & 0.34 \\
\hline $27 \mathrm{~A}$ & -33.627 & -75.842 & 5 Dec 2004 & 5 & 0.173 & 0.26 \\
\hline $30 \mathrm{~A}$ & -34.020 & -73.359 & 6 Dec 2004 & 5 & 1.620 & 1.62 \\
\hline
\end{tabular}

averaging results from eight (sometimes a few more) replicate measurements, each of which was measured from $0.5 \mathrm{~mL}$ of sample.

For both cruises, attenuation coefficients at nine wavelengths in the visible and near-IR were measured with a WET Laboratories ac-9 device according to the methods of Twardowski et al. (1999).

\section{c. Image processing and analysis to determine PSDs}

Filters were examined using a JEOL 5900 LV scanning electron microscope (SEM) in order to acquire images. The SEM was set to a low vacuum mode of $20 \mathrm{~Pa}$ and backscattered electron images were produced. The accelerating voltage was set to $20 \mathrm{kV}$ to mask fine surface structures and enhance particle edge contrast. The contrast and brightness were set to clearly distinguish the lighter shaded particles from the darker shaded background since our emphasis was on a clearly defined outline of the overall particle (Fig. 1a). For contrast, this equated to approximately $69 \%-76 \%$ full scale and for brightness to $67 \%-75 \%$ full scale. For each filter, nine random, nonoverlapping images were focused and photographed at a magnification of $1000 \times$. Images were saved as bitmap image files.

An algorithm was developed to automatically process and analyze the acquired SEM images through MATLAB in conjunction with the Image Processing Toolbox. The images were of a grayscale color map with a luminance range from 0 (black) to 255 (white). The luminance of the filter background was nearer to 0 and the luminance of the particles was nearer to 255. A histogram of the counts of each luminance value typically produced a quasi-Gaussian distribution. To distinguish the particles from the background, the shape of the histogram was analyzed. For each SEM image, a 1D median filter was applied to the image histogram, and then the differences between adjacent counts were calculated. Each SEM image was converted to a binary (black and white) image based on a threshold that was unique to each individual image using "im2bw," as described below. For each newly created binary image, the distance transform of the complement image was calculated and a Gaussian filter was applied to the distance map using "imfilter." A watershed transform using "watershed" was applied to the distance map and the watershed ridgelines separated touching particles (Fig. 1b).

An automatic thresholding protocol for each image was developed based on the image luminance histogram shape. As stated earlier, the differences in adjacent luminance counts were calculated. The luminance with the most negative difference in counts was approximately where the particles could be distinguished from the background. However, this luminance value needed some adjustment to account for the particle mass on the filter. More particles on a filter resulted in more pixels in the image being white, while fewer pixels were black such that a single threshold level could not be used for all samples. The root-mean-square error (RMSE), 


$$
\mathrm{RMSE}=\left\{\frac{\sum_{i=1}^{n}\left[\log \left(\frac{d N}{d D}\right)_{\mathrm{SEM}, i}-\log \left(\frac{d N}{d D}\right)_{\text {Coulter Counter }, i}\right]^{2}}{n-1},\right.
$$

in the residuals of Arizona Test Dust PSDs determined from SEM compared to the PSD provided by the manufacturer using a Coulter Counter was minimized to extract the best luminance adjustment per amount of material present on a filter where $i$ represents the particle diameter size bin. Once the luminance adjustment to distinguish particles from the background was determined, it was scaled to threshold level from 0 (black) to 1.0 (white) and applied to the algorithm. A power-law relationship existed between luminance adjustment and the particle mass on the filter (Fig. 2). Knowledge of mass loading prior to image processing was thus useful in removing subjectivity in the analysis, enabling full automation of luminance adjustment.

For each new processed binary image, the areas of the particles $(A)$ were analyzed using MATLAB "regionprops." Each SEM image contained a scale bar of a certain number of pixels, where, in this case, 50 pixels equaled a known distance of $10 \mu \mathrm{m}$. Particles of all sizes and shapes were included in the analysis and particles that touched the border of the images were excluded in the analysis. Particle diameters $(D)$ were calculated from the areas by assuming equivalent spherical diameter

$$
D=2 \sqrt{\frac{A}{\pi}}
$$

A histogram of the particle diameter data for each image was compiled with the bin range comprising a logarithmically spaced distribution of diameters ranging from approximately 0.40 to $20.00 \mu \mathrm{m}$ (note the $0.4-\mu \mathrm{m}$ pore size of Poretics filters). Particles consisting of seven total pixels (particle area of $0.28 \mu \mathrm{m}^{2}$ ) or less have an equivalent spherical diameter of approximately $0.6 \mu \mathrm{m}$ and are not reported in PSDs. These particles are similar in size to the filter pore size, which may contaminate this size bin (Fig. 1b). Particle frequencies $(\nu)$, the number of particles within a size bin, were summed from all nine images for each particle diameter bin and then normalized to the volume of water filtered $\left(V_{\text {filt }}\right)$ multiplied by a volume correction (see below) to calculate the number of particles in a size bin per unit volume $(d N)$,

$$
d N=\frac{\nu}{V_{\text {filt }} V_{\text {corr }}}
$$

The volume correction factor $V_{\text {corr }}$ was necessary because only a portion of the filter was imaged. The area of one rectangular image $A_{i}$ was $1.06 \times 10^{4} \mu \mathrm{m}^{2}$. The area of the filter $A_{\text {filt }}$ where particles were collected was $9.62 \times 10^{8} \mu \mathrm{m}^{2}$, which is the circular portion of the filter within the gasket of the filtration apparatus that contained the particles. The volume correction $\left(V_{\text {corr }}\right)$ was thus

$$
V_{\text {corr }}=\frac{9 A_{i}}{A_{\text {filt }}}=\frac{9.56 \times 10^{4}}{9.62 \times 10^{8}}=9.94 \times 10^{-5} .
$$

The PSD function $f(D)$ was computed from $d N / d D$ $\left(\mathrm{m}^{-4}\right)$, where $d D$ is the size of the associated particle size bin $(\mathrm{m})$.

A commonly used model to describe $f(D)$ for natural particle populations is a power-law function, also known as a differential Junge-type model (Bader 1970; Kitchen 1977; Jonasz and Fournier 2007),

$$
f(D)=\frac{d N}{d D}=N_{o}\left(\frac{D}{D_{o}}\right)^{-\xi}
$$

where $D$ is particle diameter $(\mathrm{m}), D_{o}$ is a reference particle diameter chosen to be equal to $1 \mu \mathrm{m}$, the concentration factor $N_{o}$ is the particle differential number concentration at $D_{o}\left(\mathrm{~m}^{-4}\right)$, and $\xi$ is the nondimensional particle size distribution slope. While simple and convenient, expressing the PSD in this form may not be sufficiently representative of a natural population for some applications. However, the powerlaw model has proven to be a reasonable general model for size distributions in the ocean (Kitchen 1977; Jonasz and Fournier 2007; Buonassissi and Dierssen 2010) that lends itself well to optical inversion models because only one shape parameter must be solved in inversion. The PSD was fit performing a linear regression analysis of the log-transformed variables (Myers 1990; Neter et al. 1989).

Relative error $(E)$ was calculated to determine precision based on the number of particles counted by assuming a normal distribution of particles (Sournia 1978)

$$
E=\frac{z_{(0.05)} \sqrt{\nu}}{\nu}(100 \%)
$$



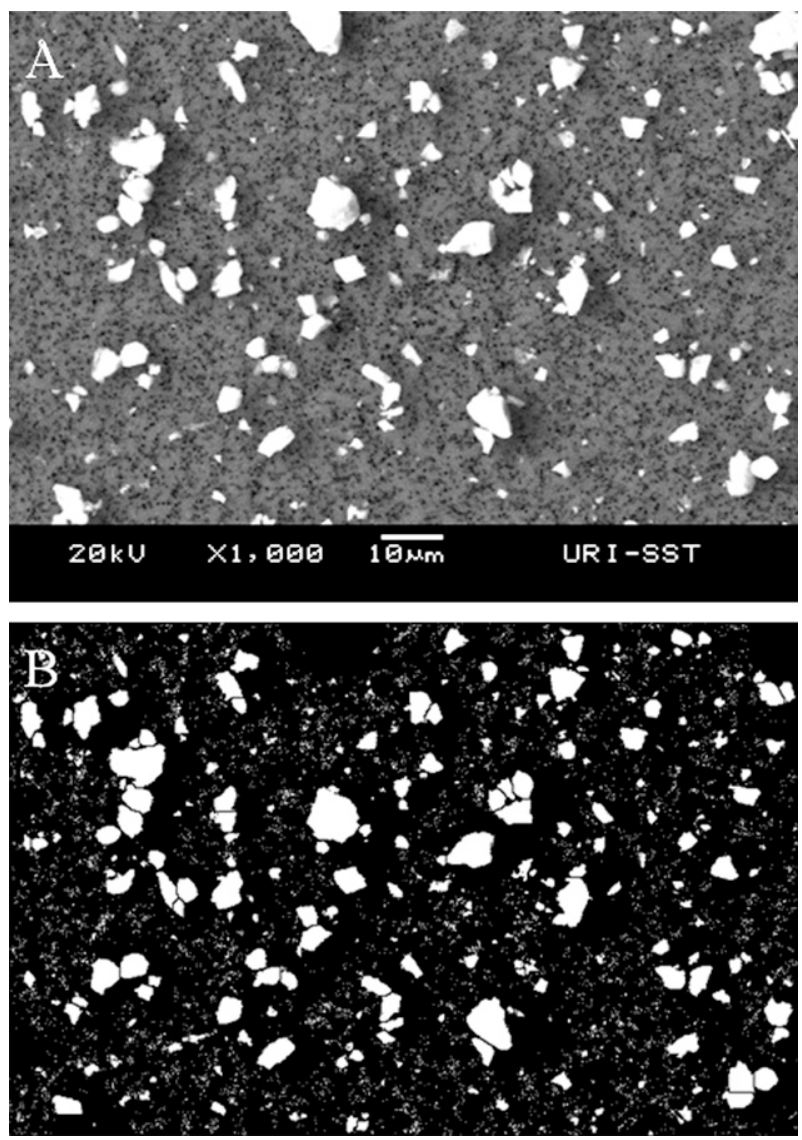

FIG. 1. (a) SEM image of Arizona Test Dust (particle mass = $0.53 \mathrm{mg}$, mass loading $=0.05 \mathrm{mg} \mathrm{cm}^{-2}$ ). (b) Image of Arizona Test Dust after automatic threshold and watershed analyses in MATLAB (see text).

where $z_{(0.05)}$ is 1.96 , which is the standard normal deviate for $95 \%$ of the area under the normal particle distribution, and $\nu$ is the number of particles per size bin for a sample.

\section{d. EDS analysis to obtain elemental distributions}

Filters were examined using a Princeton GammaTech Spirit EDS with the filter loaded in the SEM. The EDS, while interfaced with the SEM, was equipped to collect a grayscale image of the filter along with multiple binary X-ray mapping images for almost any element desired. Mapped images were collected for elements that are important in the marine environment such as but not limited to silicon, aluminum, iron, calcium, magnesium, and potassium. Carbon and oxygen could not be resolved because they were contained in the filter itself. The filter was scanned for multiple trials at 25, 40, 50, and 100 frames (complete image scans) with a scan size of $512 \times$ 384 pixels and a resolution of $2.5 \times 10^{-7} \mathrm{~m}$ per pixel. The grayscale EDS image for each trial was converted to

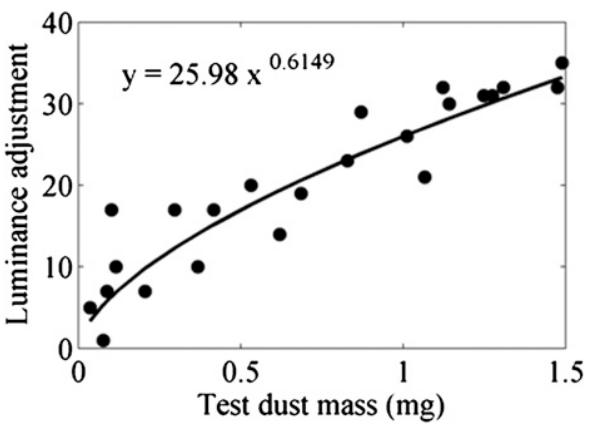

FIG. 2. Power-law relationship between the luminance adjustment for the automatic threshold method and test dust mass present on the filter. The luminance values were determined by minimizing the error of the residuals between the PSD provided by the manufacturer and the PSD determined by SEM analysis.

a binary image through a manner similar to the SEM grayscale images. PSD was determined from both the EDS binary image and the SEM image. An algorithm was developed to obtain chemical distributions of particles from the EDS elemental mapping image in reference to the binary image. Particles in the binary image were labeled using MATLAB "bwlabel" and the coordinates of those particle pixels were determined. The coordinates of pixels for the mapped elemental images were also determined. For each mapped elemental pixel coordinate that matched a binary particle pixel coordinate, the labeled binary particle was tagged and the PSD of all tagged particles for each element mapped was determined following the previously stated methods. Particle mass $(M, \mathrm{mg})$ was also computed for all particles using the density $(\rho)$ of ATD $(3.13 \times$ $10^{-9} \mathrm{mg} \mu \mathrm{m}^{-3}$ ) calculated from the individual mineral densities and their percent,

$$
M=\rho V=3.13 \times 10^{-9}\left[\frac{4}{3} \pi\left(\frac{D}{2}\right)^{3}\right]=1.64 \times 10^{-9} D^{3} .
$$

Particle mass was also calculated for particles containing a particular element using the individual density of the mineral component. For instance, for particles containing calcium, the density of calcium oxide $\left(3.35 \times 10^{-9}\right.$ $\mathrm{mg} \mu \mathrm{m}^{-3}$ ) was used in Eq. (7). The chemical composition by percent weight (wt $\%)$ for each element was calculated as

$$
\mathrm{wt} \%=\frac{M_{x}}{M_{\text {total }}}(100 \%)
$$

where $M_{x}$ is the sum of the mass of particles containing a particular element $(x)$ and $M_{\text {total }}$ is the summed mass of all particles from the corresponding binary image. 


\section{Results}

\section{a. Comparison and validation}

\section{1) Arizona Test Dust Particle Size DISTRIBUTION}

Twenty-four subsamples of a prepared suspension of ATD were analyzed for PSD, with the subsamples covering a range of $0.04-1.49 \mathrm{mg}$ in particle mass on the filter. PSDs determined from SEM analyses agreed well with the PSD provided by the manufacturer using a Coulter Counter (Fig. 3). This is not surprising because thresholding was optimized to match the Coulter Counter PSD. Relative errors were calculated from the number of particles counted per size bin (Fig. 3). Errors per size bin were in excess of $25 \%$ over most sizes for samples that had lower particle masses (Fig. 3a) while errors were around $10 \%$ for particle sizes less than $2 \mu \mathrm{m}$, increasing to $30 \%$ for particle sizes of $2-6 \mu \mathrm{m}$ for samples with particle masses greater than $0.5 \mathrm{mg}$ (Figs. 3b,c). This suggested that there was a sufficient amount of particles present to be counted to give precise results for smaller particle sizes as long as the particle mass on the filter exceeded about $0.5 \mathrm{mg}$. Precision within the larger particle size measurements could be improved by analyzing more than nine random SEM images. The PSDs of Arizona Test Dust from Coulter Counter analysis and SEM analysis were modeled well with a power law [Eq. (5)] for particle diameters between 0.72 and $3.09 \mu \mathrm{m}$ when $M$ was around $0.5 \mathrm{mg}$, with a $\xi$ of approximately 2 with $R^{2}$ values ranging from 0.87 to 0.98 . The distributions did not match well for particle masses on the filter for the smallest (Fig. 3a) and largest (Fig. 3c) ends of the particle mass range tested, and $\xi$ did not agree well with the PSD provided by the manufacturer for these cases.

Clearly, particle density on the filter substantially impacted PSD assessments. TSM analysis of the test dust showed a linear relationship between the mass of test dust on the filter as a function of the volume of suspension filtered (Fig. 4a). The linear fitted TSM of the prepared test dust suspension was $7.38 \mathrm{~g} \mathrm{~m}^{-3}$. Slopes $\xi$ were calculated for the PSDs of all test dust filters for particle diameters between 0.72 and $3.09 \mu \mathrm{m}$, and percent errors relative to the Coulter Counter estimate were within $20 \%$ when the mass of test dust on the filter ranged between about 0.4 and $1.0 \mathrm{mg}$ (Fig. 4b). For the filters with minimal test dust mass, there tended to be a positive error, and for the filters with greater test dust mass, there tended to be a negative error. The positive percent error could be a result of either an overestimation of smaller particles or an underestimation of larger particles. The latter was most likely the case with larger particles being underrepresented on the filters with minimal
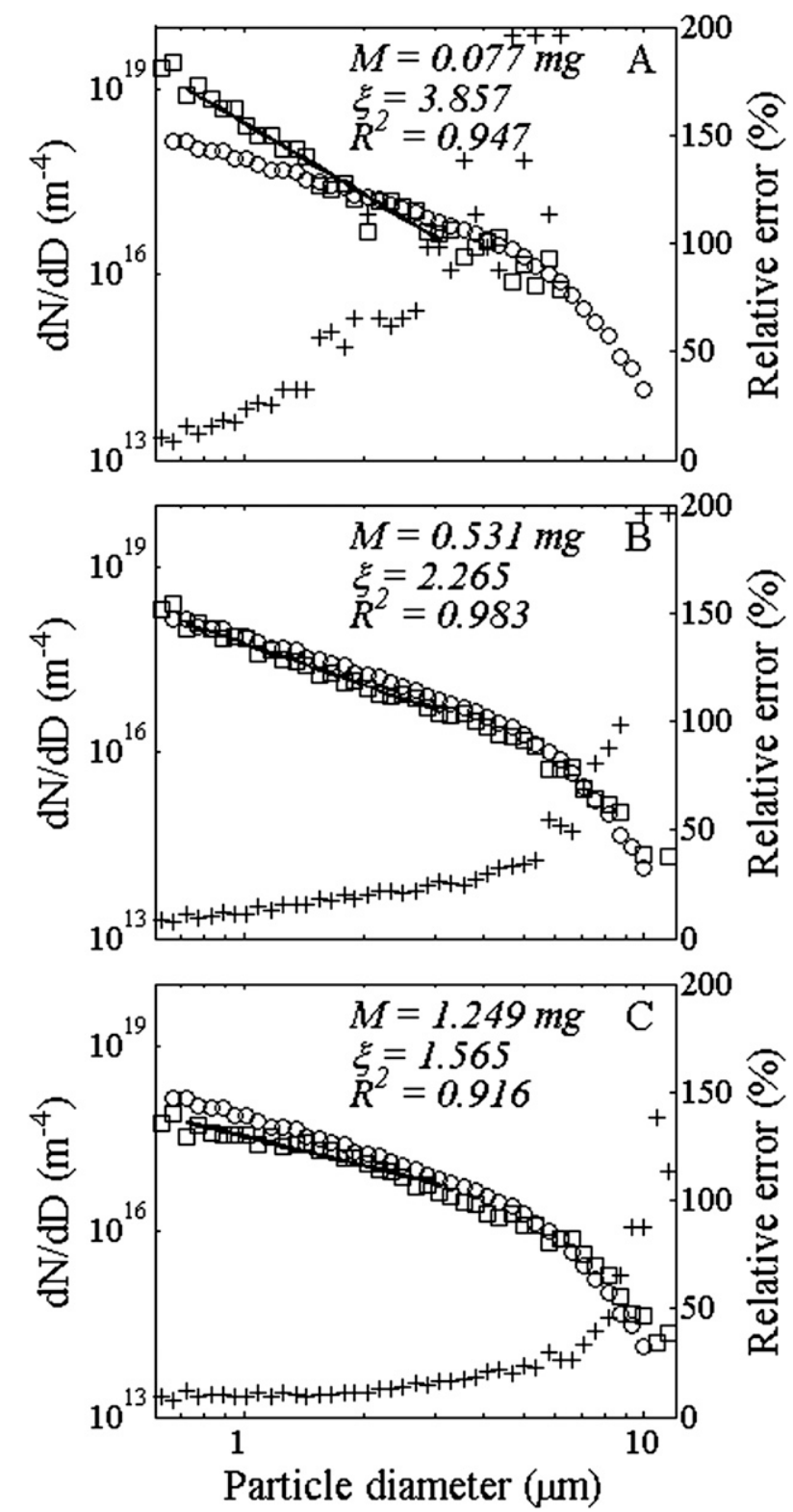

FIG. 3. PSDs of an Arizona Test Dust suspension with (a)-(c) three subsamples shown out of 24 different filter masses analyzed. SEM analyses are shown (squares) overlapping Coulter counter PSDs (circles) and SEM relative errors calculated from the number of particles counted per size bin shown as pluses. In the upper-right corner of each panel is the particle mass $(M)$ on the filter. Power trend lines were calculated for particle diameter sizes of 0.72 $3.09 \mu \mathrm{m}$ for PSDs determined from SEM analysis (solid lines).

test dust mass as shown by the relative errors (Fig. 3a). The negative percent errors for higher test dust masses is likely due to shadows created around larger particles that can effectively hide small particles. Particle overlap also became a problem at high concentrations when the automatic watershed processing in some cases combined two or more particles into one larger particle. 

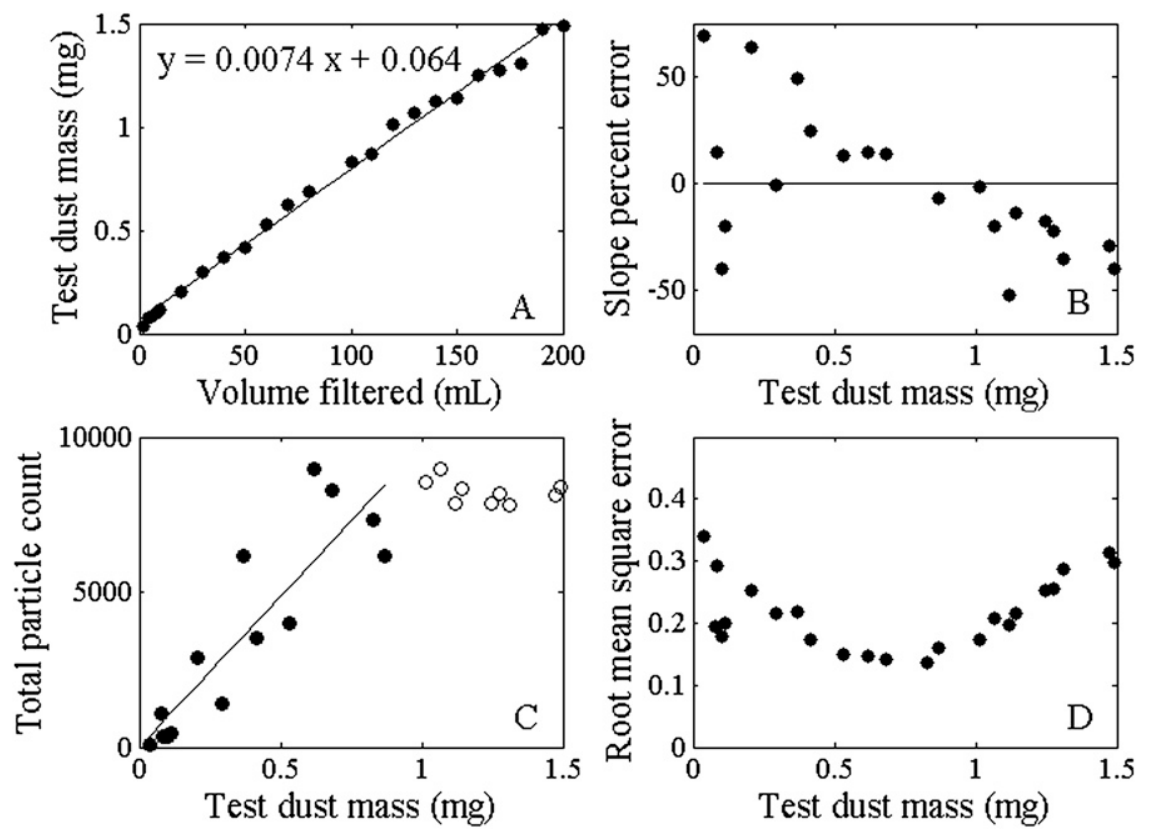

FIG. 4. (a) Test dust mass $(M)$ on the filter as a function of volume of test dust suspension filtered $\left(V_{\text {filt }}\right)$. The linear relationship indicates the TSM of the suspension is approximately $7.4 \mathrm{~g} \mathrm{~m}^{-3}\left(R^{2}=0.99\right)$. (b) Percent errors of the test dust slope $(\xi)$ determined from SEM compared to the slope provided by the manufacturer as a function of test dust mass on the filter. Uncertainties in the slope determined from SEM analysis increase as the mass of material present on the filter is below 0.4 or above $1.0 \mathrm{mg}$. (c) Total test dust particles counted from SEM as a function of test dust mass on the filter. When the mass of material present on the filter exceeds approximately $0.8-1.0 \mathrm{mg}$, it is difficult to resolve the number of particles for a sample $\left(R^{2}=0.7867\right)$. (d) RMSE between PSDs determined from SEM analysis and Coulter Counter as a function of test dust mass on the filter. Best agreements (smallest errors) are found when the mass of material present on the filter is between 0.4 and $1.0 \mathrm{mg}$.

The total number of test dust particles counted on the filters displayed a linear relationship with test dust mass on the filter up to masses of approximately $1.0 \mathrm{mg}$ (Fig. $4 \mathrm{c} ; R^{2}=0.99$ ). The leveling off of particle counts after test dust masses greater than $1.0 \mathrm{mg}$ is attributed to particle shadowing and overlap. RMSE displayed a trend with test dust mass on the filter (Fig. 4d). The lowest error, or best agreement, occurred for samples with test dust masses of between 0.4 and $1.0 \mathrm{mg}$. These results suggested the amount of material loaded onto a filter should be within about 0.04 and $0.1 \mathrm{mg} \mathrm{cm}^{-2}$ to optimize particle dispersion and visible separation of the particle field on the filter.

For the most part, the PSD method presented here is fully automated with the exception of acquiring the SEM images. The current method places significant emphasis on the accuracy of the Coulter Counter PSD provided by the manufacturer, because some reference is desirable for automating threshold levels. The manufacturer uses a dilute suspension and a surfactant to avoid any aggregation (PTI 2006, personal communication), ATD is not electrolytic, and the distribution is well constrained to $<10 \mu \mathrm{m}$, so that, most of the reported drawbacks to using a Coulter Counter do not apply here. The close agreement of the reported distribution $<4 \mu \mathrm{m}$ (the size range that is not affected by the manufacturer's mechanical separation technique) with the theoretically predicted and experimentally observed Junge-type model (Junge 1963; Bader 1970) lends credibility to the data. Setting the threshold for each image is derived from the power-law relationship between the amount of material on the filter and the luminance adjustment (Fig. 2). Other options are possible, including setting a constant threshold (Allen 1997), although the method here is expected to be more accurate. Future modifications to the method might include an automated sensitivity analysis where different threshold values are compared to determine when the largest errors are introduced into the analysis.

\section{2) Arizona Test Dust particle Chemical COMPOSITION}

ATD PSDs for particles containing silicon, aluminum, or iron determined from EDS roughly followed a power-law 

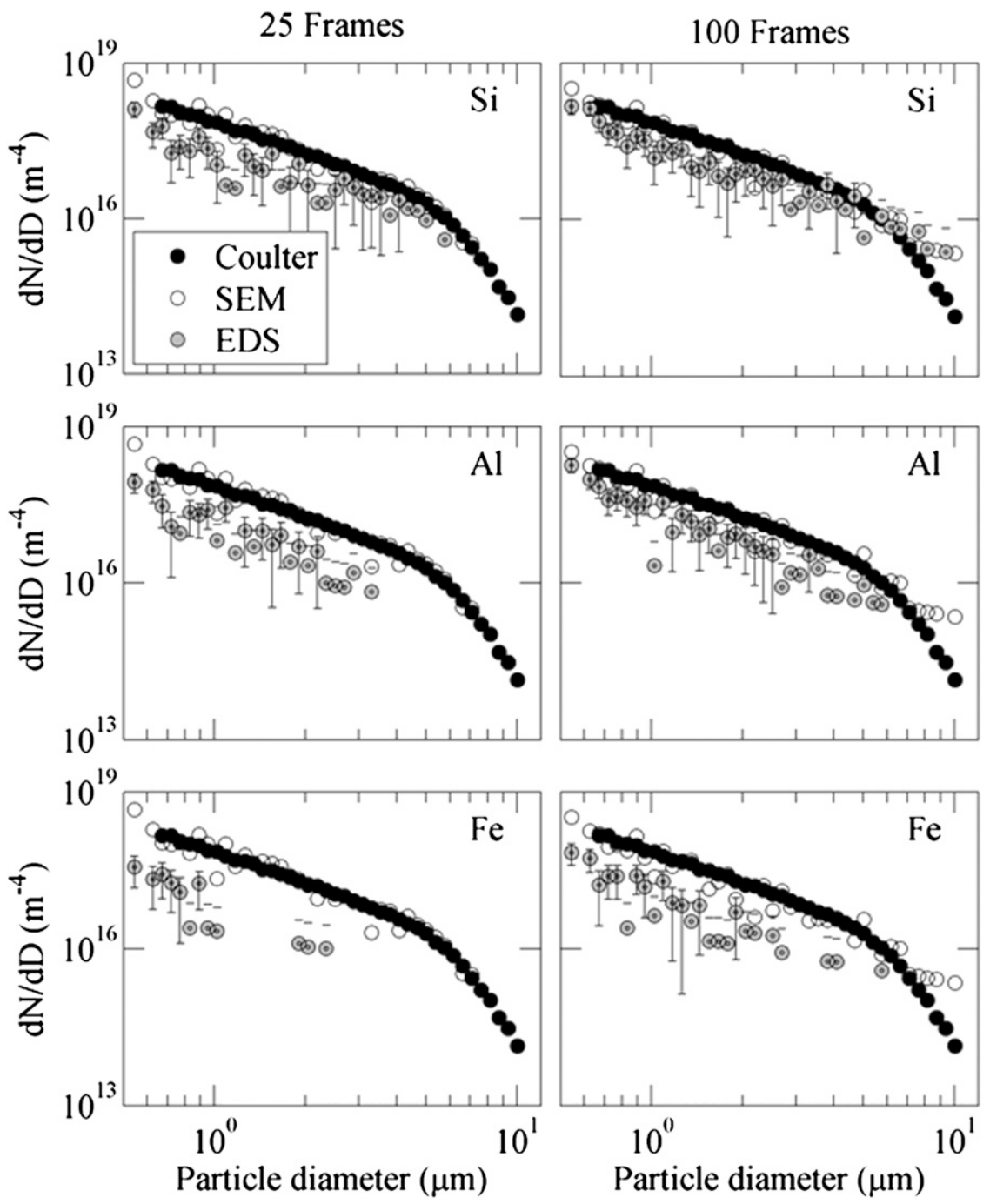

FIG. 5. Silicon, aluminum, and iron compositions (gray circles) determined from EDS analysis with respect to particle size for Arizona Test Dust. For reference, the PSD determined from SEM (open circles) and Coulter Counter (black points) are also plotted. Results from (left) 25 mapped frames and (right) 100 mapped frames. The higher number of mapped frames yielded less noise in the analysis. The filter contained $0.53-\mathrm{mg}$ material.

model (Fig. 5) and were within $20 \%$ of the slope that was estimated for the entire particle population (data not shown). Left-hand-side panels show compositions determined from 25 mapped frames (image scans) and righthand-side panels from 100 mapped frames. As the number of frames mapped increased, the more particles were tagged with concurrent reduction in statistical noise. Chemical composition PSDs determined from EDS are noisy with respect to the SEM-imaged PSDs because only one image was analyzed for the former versus the sum of nine images for the latter. Collection of more than one image per filter would improve counting statistics, although instrument time needs to be considered.
The time required to map 25 frames of a filter was approximately $20 \mathrm{~min}$ while 100 frames required approximately $90 \mathrm{~min}$. Chemical compositions determined from EDS analyses using 100 frames agreed well with the chemical compositions provided by the manufacturer (Table 2). It is noted that aluminum is slightly higher, and this is most likely due to contamination from an aluminum ring that that is used as a weight to hold the filter in place while loaded in the SEM. At this time, chemical compositions by percent weight cannot be determined for field samples because particle densities will not be known a priori and vary over wide distributions. However, the concentration distributions 
TABLE 2. Composition of Arizona Test Dust by percent weight calculated from 100 mapped frames for EDS analysis.

\begin{tabular}{lcr}
\hline \hline & \multicolumn{2}{c}{ Chemical composition (\% weight) } \\
\cline { 2 - 3 } Chemical & Manufacturer & EDS \\
\hline $\mathrm{SiO}_{2}$ & $68-76$ & 66.9 \\
$\mathrm{Al}_{2} \mathrm{O}_{3}$ & $10-15$ & 21.9 \\
$\mathrm{Fe}_{2} \mathrm{O}_{3}$ & $2-5$ & 4.2 \\
$\mathrm{CaO}$ & $2-5$ & 3.7 \\
$\mathrm{MgO}$ & $1-2$ & 4.3 \\
$\mathrm{TiO}_{2}$ & $0.5-1.0$ & 2.2 \\
$\mathrm{~K}_{2} \mathrm{O}$ & $2-5$ & 2.7 \\
\hline
\end{tabular}

of element-containing particles in a sample can be assessed with EDS analysis providing useful information for many geochemical, geophysical, and optical applications.

\section{b. Coastal water case}

\section{LONG ISLAND SOUND}

PSDs determined from the Elzone particle analyzer with $30-$ and $120-\mu \mathrm{m}$ orifices, the submersible LISST100X laser diffraction sensor, and the SEM method showed reasonable agreement (Fig. 6). Relative errors for SEM were around $20 \%$ for particles with diameters $<6 \mu \mathrm{m}$ and then increased for larger particles because there were fewer particles counted. All PSDs were approximately linear on a log- $\log$ scale in the $2-10-\mu \mathrm{m}$ size range, indicating that the Junge-type power-law relationship provided a reasonable description of the general distribution of the data. Power-law slopes $\xi$ were calculated over this size range resulting in $R^{2}$ values from 0.74 to 0.98 . It is duly noted that there is no a priori expectation that PSDs from natural samples should necessarily
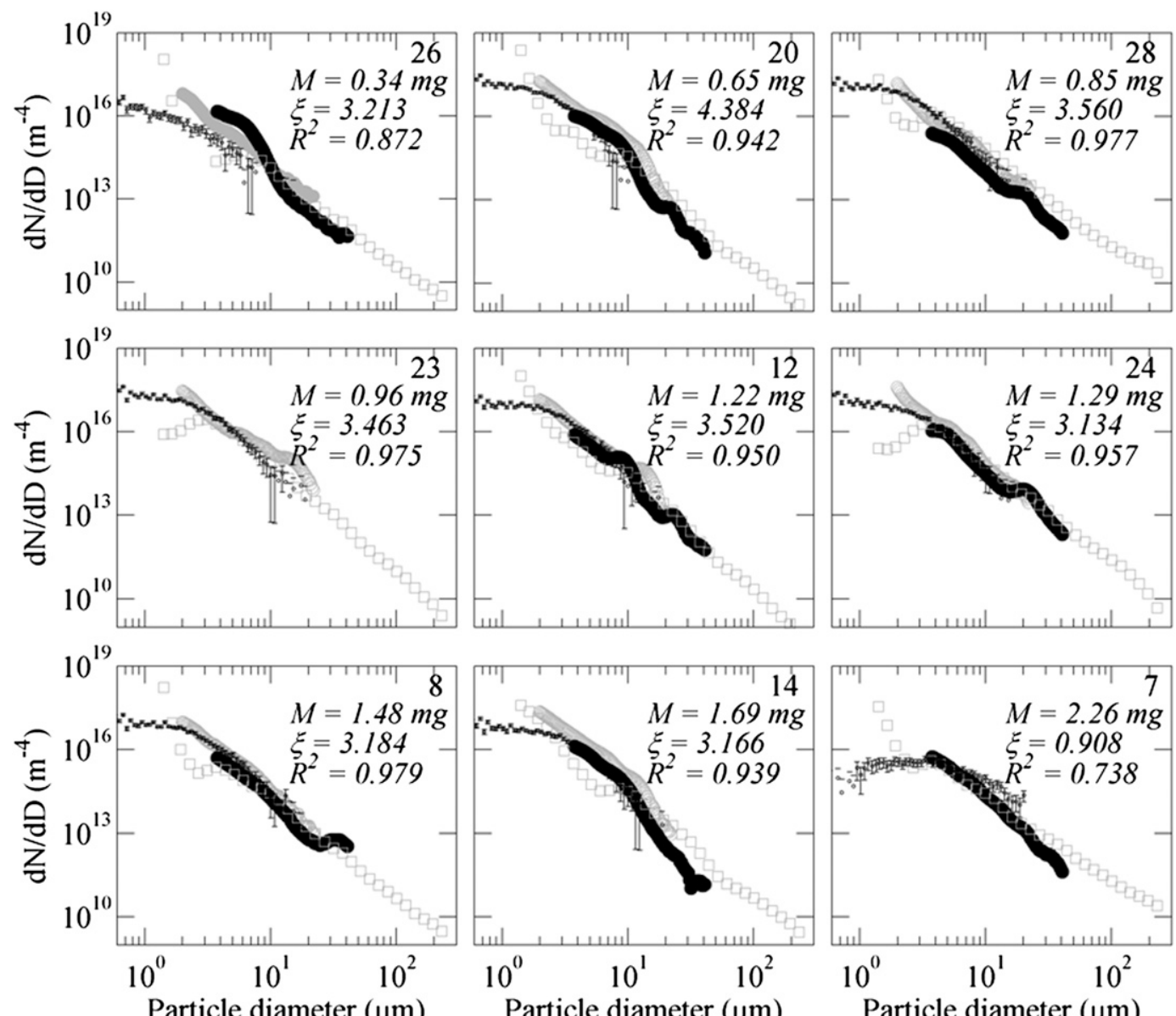

FIG. 6. Particle size distributions determined from SEM (small filled squares, with error bars), LISST-100X (gray open squares), $30-\mu \mathrm{m}$ orifice Elzone (gray open circles), and $120-\mu \mathrm{m}$ orifice Elzone (black filled circles) for Long Island Sound samples. The numbers in the upper right corner of each panel are the station identification, the particle mass $(M)$ on the filter $(\mathrm{mg})$, modeled slope $(\xi)$ from SEM, and its respective correlation coefficient $\left(R^{2}\right)$. The steep "s" shape observed in the LISST-100X data at small size classes is likely a methodological artifact (Buonassissi and Dierssen 2010; Reynolds et al. 2010). 
be modeled well with a power fit. It is used here as a broad estimator of PSD shape, similar to a mean size parameter. Inconsistencies or consistencies with a power fit are noted only as a reference point in comparisons. There were several relatively small deviations from a power-law model that were sometimes observed in one or more of the PSD estimates, although most were unique to a particular PSD method, which therefore lowered confidence in the reality of the features. An example is the Elzone PSD with $120-\mu \mathrm{m}$ orifice for station 26 (Fig. 6, upper left panel). Values of $d N / d D$ for particles $<10 \mu \mathrm{m}$ were higher than values from the other methods. For stations 28 (Fig. 6, upper right panel) and 24 (Fig. 6, middle right panel), Elzone PSDs with the 30- and $120-\mu \mathrm{m}$ orifices both appeared to show a small enhancement above a power-law approximation around $11 \mu \mathrm{m}$, but this was not observed by the LISST-100X. Given the uncertainties in the assumptions used to process PSD data (e.g., spherical particles) and the complexities of the Long Island Sound particle assemblages (Aurin et al. 2010), such minor features may be measurement artifacts.

There were also consistent features to the PSD shapes for the different methods, particularly for particles in the smaller end of the size range. PSDs with the Elzone with the $30-\mu \mathrm{m}$ orifice generally showed an increase above a power-law approximation for particles smaller than about $3 \mu \mathrm{m}$ that was not observed with other methods. The SEM method conversely displayed a decrease relative to a power law for particles smaller than about $2 \mu \mathrm{m}$. Relative to a power law, the LISST-100X PSDs showed a consistent minimum around $2 \mu \mathrm{m}$ and then a rapid increase for smaller particles, neither of which were observed by the other methods. The tail in the PSD spectrum from the LISST-100X is likely an artifact of the measurement technique that has been observed by others (Buonassissi and Dierssen 2010; Reynolds et al. 2010). It was clear from the Arizona Test Dust analyses that filter particle masses greater than about $1.0 \mathrm{mg}$ started to present problems with the underestimation of small particles resulting from shadowing and overlap; this was likely the case for stations $12,24,8,14$, and 7 . Note that these data from the field were collected before the analysis of optimal filter mass was carried out. However, the same effect was observed for other stations with lower filter masses also, suggesting that the flattening of the PSD at the small particle end of the distribution may be a legitimate observation. Indeed, the limited number of previous studies that have resolved particles in this size range have observed a similar flattening (Longhurst et al. 1992; Wells and Goldberg 1992; Vaillancourt and Balch 2000). The PSD for the Elzone with the $30-\mu \mathrm{m}$ orifice showed a relative increase for small particles for most of the stations that was not observed by the other methods. Others previously have noted an apparent artificial increase for the smallest particles for electroresistance-type devices (Jackson et al. 1997). At present, however, no single method can be considered optimal, particularly for complex coastal waters like Long Island Sound.

Power-law slopes $\xi$ were calculated over the common size range resolved by all methods $(2-10 \mu \mathrm{m})$ and plotted with respect to particle mass on the filter (Fig. 7a), beam attenuation coefficient at $650 \mathrm{~nm}\left(c_{650}\right)$ (Fig. $7 \mathrm{~b}$ ), and TSM (Fig. 7c). Slopes were typically between 3 and 4 , consistent with results from the literature for the coastal ocean (Kitchen 1977; Jonasz and Fournier 2007). Note that natural variability may be expected among these samples and the worst slope deviation for SEM had the highest filter mass. Overall, the slopes obtained with the LISST-100X were typically lower than the slopes from SEM and Elzone with the $30-\mu \mathrm{m}$ orifice, likely because of the relative decrease in the PSD distributions at smaller particle sizes. There was generally good agreement between slopes from SEM and Elzone with the 30$\mu \mathrm{m}$ orifice except for two samples, even though many of the Long Island Sound samples analyzed had particle masses greater than $1.0 \mathrm{mg}$.

\section{c. Open ocean case}

\section{SOUTH PACIFIC GYRE}

PSDs from the South Pacific samples determined from the Beckman Coulter Counter and the SEM method mostly showed fair agreement (Fig. 8), with typically very good agreement for samples with particle masses on the filter between about 0.3 and $1 \mathrm{mg}$. Relative errors for SEM were around $15 \%$ for particles with diameters less than $2 \mu \mathrm{m}$, increasing for larger particles. Power-law slopes $\xi$ were calculated over a size range of $0.77-10 \mu \mathrm{m}$, with $R^{2}$ values from 0.92 to 0.98. Sample 30A (Fig. 8, lower left panel) has more than $1 \mathrm{mg}$ of particle mass on the filter, and the PSD did not agree well with the Beckman Coulter distribution. PSDs from the Beckman Coulter always showed rapid increases in the distribution for particles with diameters in the range of $0.6-1 \mu \mathrm{m}$, similar to the Elzone with the $30-\mu \mathrm{m}$ orifice, which are not apparent in the PSDs from SEM.

Power-law slopes $\xi$ were calculated over the common size range resolved by both methods $(1-10 \mu \mathrm{m})$ and plotted with respect to the particle mass on the filter (Fig. 9a), the beam attenuation coefficient at $650 \mathrm{~nm}$ $\left(c_{650}\right)$ (Fig. 9b), and TSM (Fig. 9c). There was no obvious relationship between PSD slopes and mass on filter $c_{650}$ and TSM, with slopes again falling between 3 and 4 . 

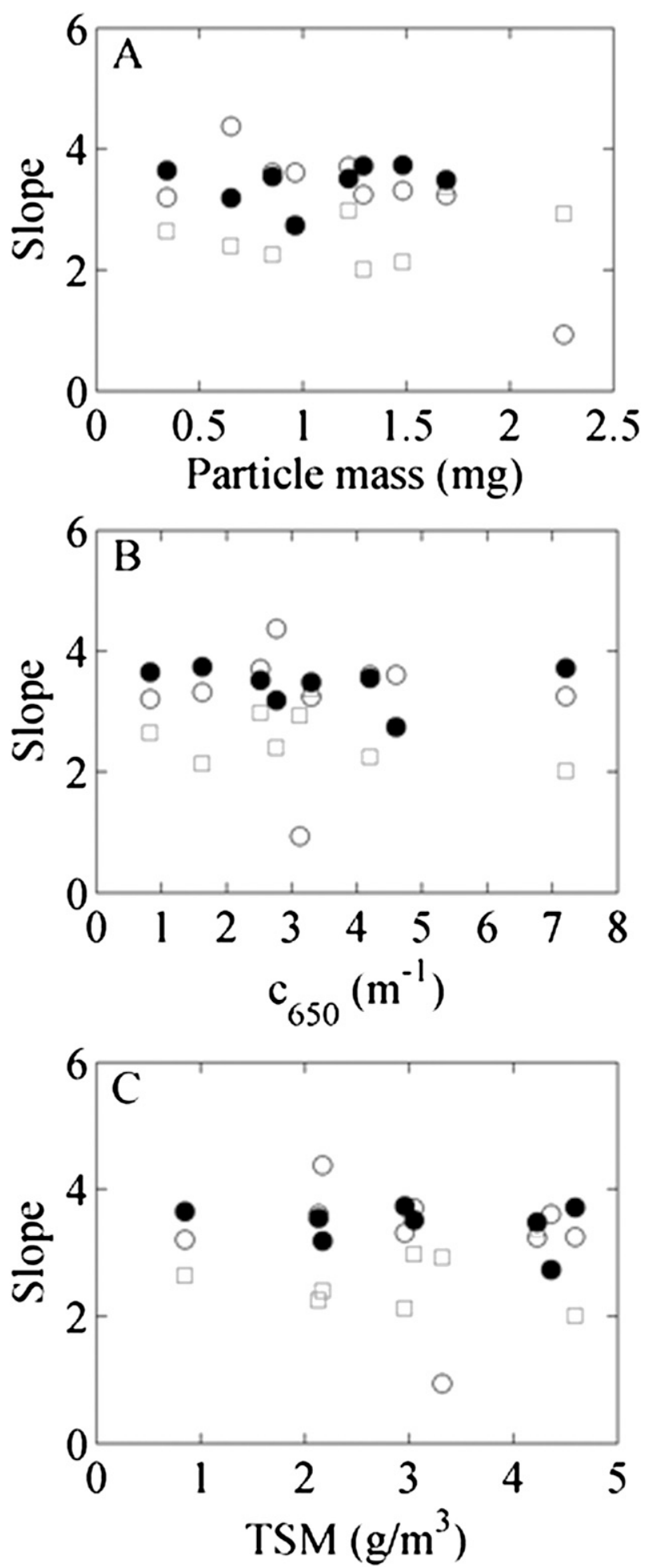

FIG. 7. Comparison of slopes calculated for particle diameters from 2 to approximately $10 \mu \mathrm{m}$ from SEM analysis (open circles), LISST-100X (open squares), and 30- $\mu \mathrm{m}$ Elzone (black filled circles) for Long Island Sound samples as a function of mass on (a) the filter, (b) $c_{650}$, and (c) TSM.
Particle concentrations from these very clear South Pacific waters were 1-2 orders of magnitude lower than those in Long Island Sound. Compositional differences could also be clearly seen in representative SEM images (Fig. 10). The Long Island Sound sample was dominated by suspended sediment with some diatom frustule fragments, while in the particle population from the South Pacific there were numerous coccolithophore plates. Particles such as these plates with marked nonsphericity may in part have caused some of the differences between the Coulter Counter and the SEM.

\section{d. Optimizing sample collection}

Our presented method for determining PSDs has an optimal range for the mass of particulate material on the filter of $0.4-1.0 \mathrm{mg}$, which is difficult to assess in the field. We investigated methods to determine filter volumes in the field to ensure that the filter particle mass fell in this range. For the Long Island Sound samples there was a roughly linear relationship between the beam attenuation coefficient at $650 \mathrm{~nm}\left(c_{650}\right)$ and TSM, with a slope close to 1 (Fig. 11). This slope is generally consistent with results from previously published works in the coastal and open ocean (Gardner et al. 2001; Babin et al. 2003; Boss et al. 2009). Since $c_{650}$ is an easily measured parameter in the field with real-time data access, it is possible to use this relationship to quickly estimate appropriate filtering volumes $(V)$, keeping in mind that an optimal particle mass on the filter $(M)$ would be around $0.7 \mathrm{mg}$ (see Fig. 4d) and that the slope $S$ of the relationship between $c_{650}$ and TSM was approximately $1 \mathrm{~m}^{2} \mathrm{mg}^{-1}$ :

$$
V \approx \frac{M}{c_{650} S} \approx \frac{0.7}{c_{650}}
$$

\section{Discussion}

A method for obtaining PSDs and particle chemical composition distributions was developed using SEM in conjunction with EDS, which removes user bias in processing images, effectively automates the method, and provides guidance in field sampling to optimize the accuracy of the results. This work builds on previous SEMbased PSD studies by also comparing results with other conventional particle sizing techniques in regions of the PSD where sizes overlapped. Additional novel aspects of method development were verification against a standard reference sample with a well-documented distribution and composition, and effective avoidance of chemical treatment of the sample beforehand.

A significant impetus for developing this technique was to improve our ability to resolve particle sizes less 

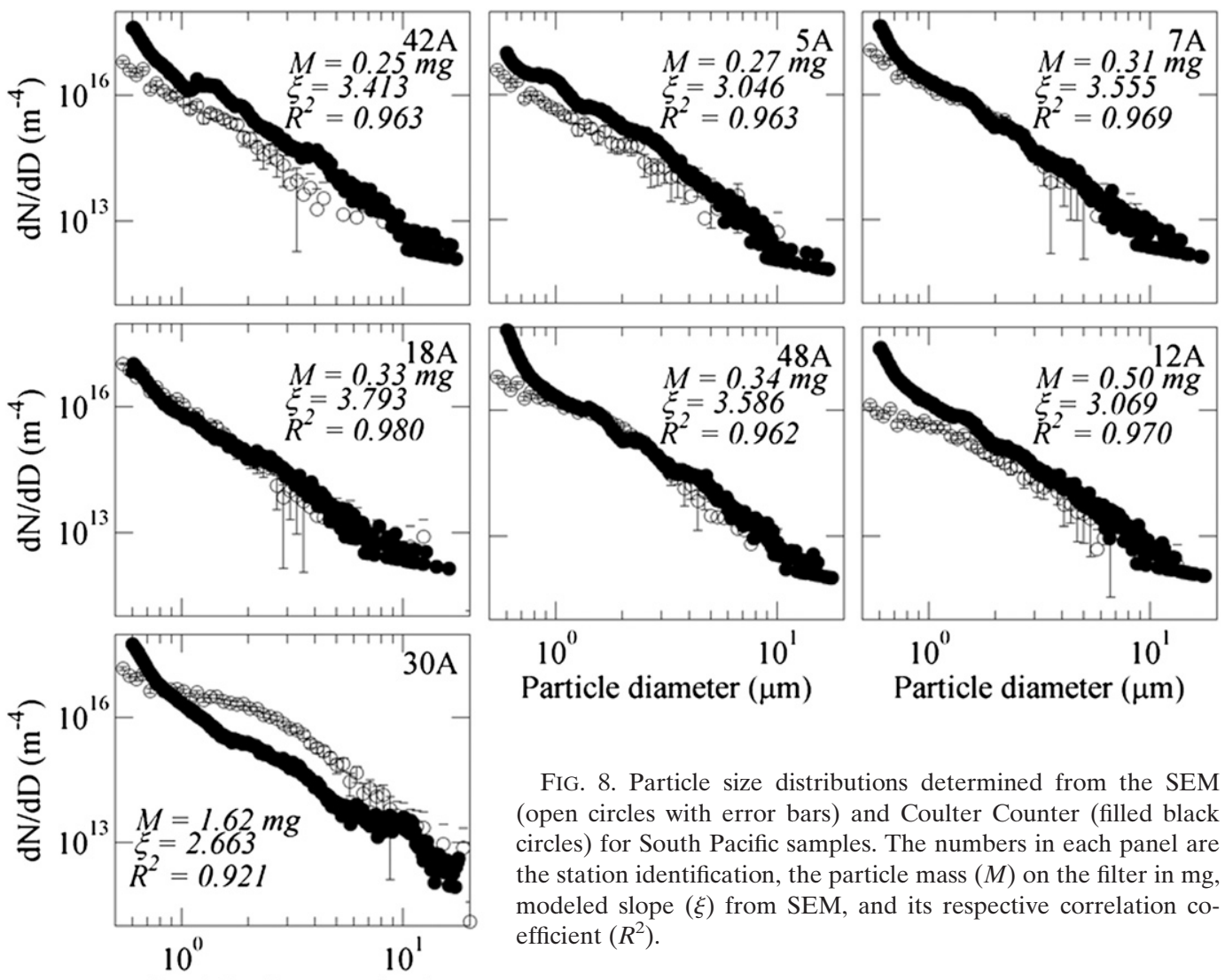

FIG. 8. Particle size distributions determined from the SEM (open circles with error bars) and Coulter Counter (filled black circles) for South Pacific samples. The numbers in each panel are the station identification, the particle mass $(M)$ on the filter in $\mathrm{mg}$, modeled slope $(\xi)$ from SEM, and its respective correlation coefficient $\left(R^{2}\right)$.

Particle diameter $(\mu \mathrm{m})$

than 1-2 $\mu \mathrm{m}$. These small particles significantly impact optical scattering in aquatic environments (Stramski and Kiefer 1991; Stramski et al. 2004; Twardowski et al. 2007), although their distributions remain poorly understood because methods to accurately enumerate them are lacking, limited, or laborious. Because these small sizes straddle the lower limit for electroresistive and diffractometry techniques, SEM or TEM may be considered the only reliable apparatus at this time for making these determinations. Electroresistive sensors have untenable clogging problems using the smallest orifices except in the very clearest natural waters. Coincidence errors are also higher for the smallest particles. Laser diffraction methods reach the diffraction limit in resolving the nearforward-scattering field when particle sizes approach about 3-4 times the wavelength of light of the source beam. Field-flow fractionation (FFF) methods offer an interesting possibility, although results are largely qualitative to this point (Vaillancourt and Balch 2000).

Even among the few SEM and TEM analyses of oceanic PSDs reported in the literature, there is a large range in Junge-type PSD slopes, from 2.6 to 12.1, when considering size ranges less than about $2 \mu \mathrm{m}$ (Harris 1977;
Longhurst et al. 1992; Kim et al. 1995). This wide range of variability is inconsistent with the relatively narrow range in slopes (typically 3-4) observed for PSDs over ranges with minimum diameters greater than about $2 \mu \mathrm{m}$ (see Jonasz and Fournier 2007, and their Tables 5.8 and 5.9 for summary of slope data from the literature). It is difficult to intimate, at least from the experimental data, whether such wide variability in slopes of the PSD less than $2 \mu \mathrm{m}$ may be accurate or to what degree they result from possible methodological artifacts. From a theoretical perspective, size distributions of the small particles should be controlled by coagulation processes mediated by Brownian motion, with a predicted steady-state theoretical slope of 2.5 (Hunt 1980). Certainly there is much to be learned about oceanic PSDs in the smallest size fractions.

While the refinement of the SEM PSD method here appears to provide reasonable size distributions down to $0.6 \mu \mathrm{m}$, certain aspects of the technique are not optimal from an optical perspective and can introduce some error in the method. For example, particles must be obtained from discrete samples passed through a filter, which mixes the finescale structure from within the water column and may break up aggregates. With care (low 

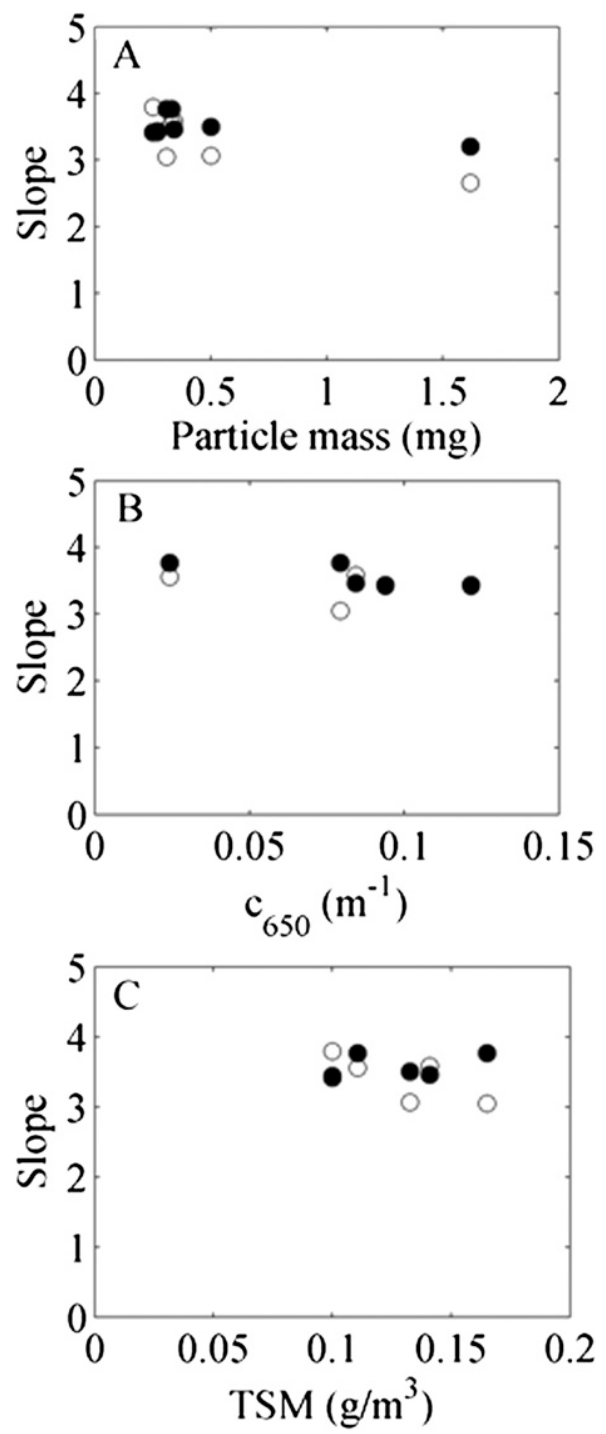

FIG. 9. Comparison of slopes calculated for particle diameters from 1 to approximately $10 \mu \mathrm{m}$ from SEM analysis (open circles) and Coulter Counter (filled black circles) for South Pacific samples as a function of mass on (a) the filter, (b) $c_{650}$, and (c) TSM.

vacuum pressure, careful handling of sample, etc.), however, disaggregation can be mostly restricted to delicate detrital particles and breaking of diatom chains and the like, as opposed to cellular disintegration. Particles also typically lie flat on filters, with their longest axis and largest cross-sectional area presented. There are algorithms for correcting the subsequent bias in sizing particles (Jonasz 1987). Finally, dehydration can shrink cellular components of the population by $\sim 10 \%$ (Jonasz and Fournier 2007), although Doucet et al. (2005) have demonstrated that SEM may be used to obtain PSDs for samples that have not been dehydrated. Bias errors may also results from dehydration of porous particle
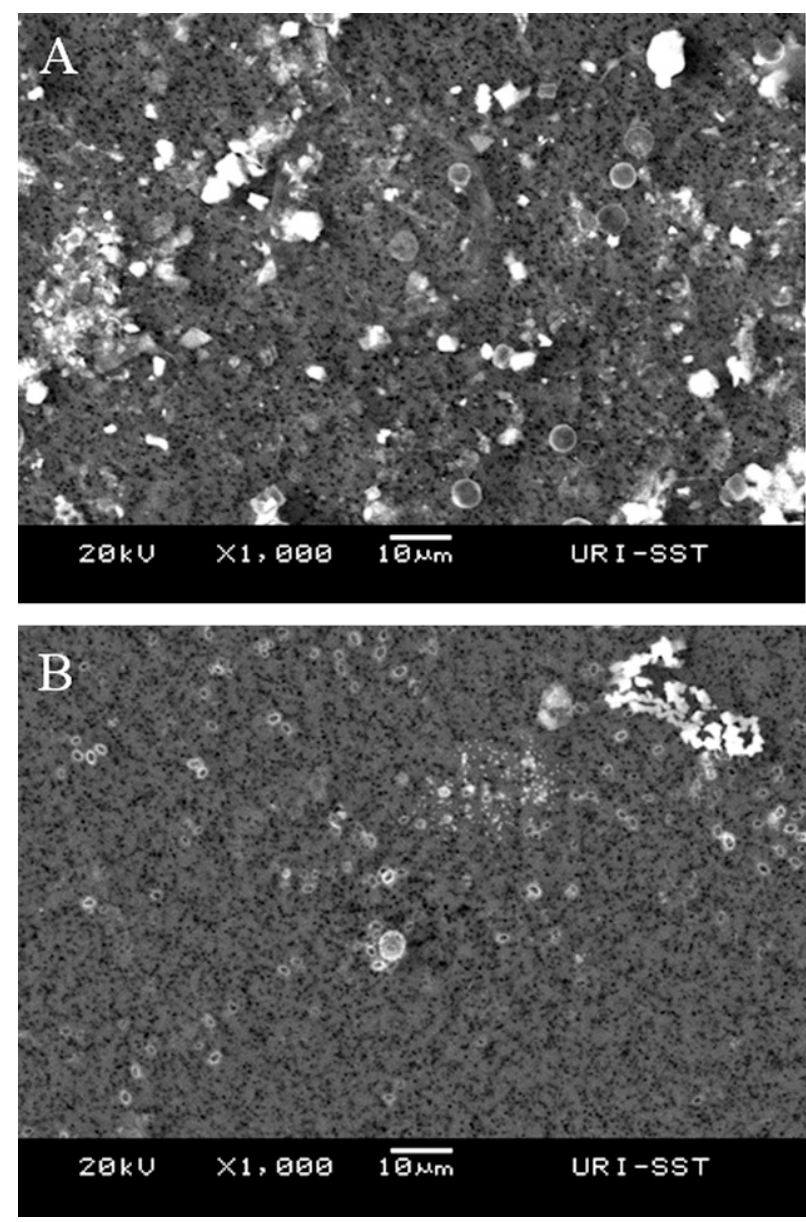

FIG. 10. (a) SEM image of Long Island Sound sample at station 28 (particle mass $=0.85 \mathrm{mg}$ ). (b) SEM image of South Pacific sample at station $12 \mathrm{~A}$ (particle mass $=0.50 \mathrm{mg}$ ).

aggregates, the extent of which will depend on the interstitial water content and composition of the aggregate. In these respects, comparison of the SEM PSD method presented here to electroresistive and laser diffraction methods is telling, because these methods, although having other biases, do not preferentially resolve the largest cross section of a particle or require dehydration. For larger particle size ranges that overlapped among these methods, agreement was generally acceptable when particle masses on the filter did not exceed $1.0 \mathrm{mg}$. This was true even for the very clear South Pacific gyre, where most of the particles (cells and organic based detritus with interstitial water) may be expected to be sensitive to dehydration.

Another motivation for employing the SEM-EDS technique for oceanic particles was to resolve elemental composition of the particles, which can be used to infer bulk particle refractive index distributions that are needed to model light scattering and study biogeochemical 


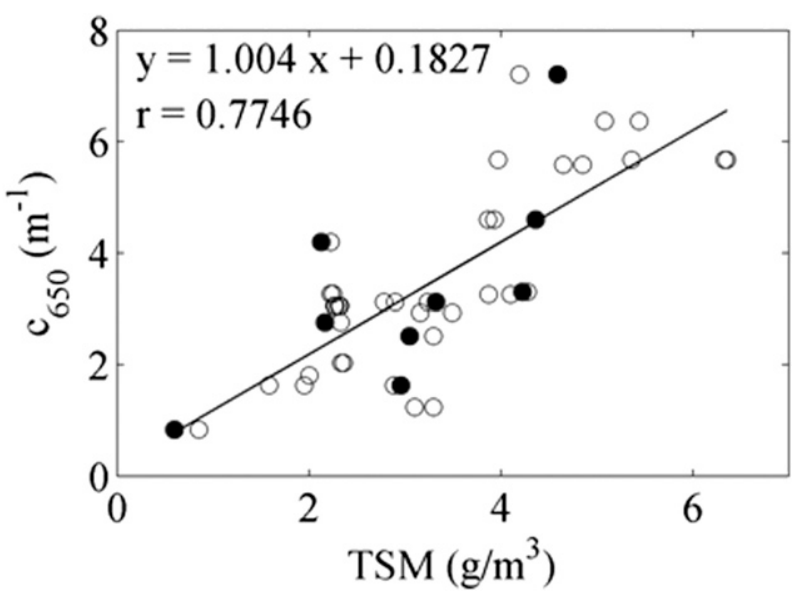

FIG. 11. Relationship between $c_{650}$ and total suspended material for Long Island Sound samples. Solid points represent samples analyzed by SEM.

cycling of elements. With distributions of elemental compositions now resolved for our reference material, the next step is to identify material compositions in natural oceanic samples in order to assign appropriate refractive indices. Incorporating automated shape analysis may also be necessary for some particles (Sosik and Olson 2007). For example, both quartz and diatom frustules are dominated by silicon and are thus difficult to distinguish based on elemental composition alone. However, their respective shapes are very different, with the former being nominally cubic or polyhedron shaped and the latter being represented by a variety of shapes, usually embedded within a porous structure. Using elemental composition as well as shape analysis may enable particle identification, effective classification, and subsequent refractive index distribution derivation in future work

There are also emerging technologies for enumerating and classifying the ocean's smallest particles that may complement the capabilities of SEM-EDS. One of the most promising is digital holographic microscopy (Sheng et al. 2006, 2007, 2008). With recent advances in digital imaging and automated reconstructive processing, as well as form factors for optical layouts that are amenable to submersion in situ (Pfitsch et al. 2005, 2007), holographic microscopy may prove to be a viable approach for imaging, sizing, and classifying undisturbed, natural particle population suspensions in the smallest size fractions in the future.

\section{Conclusions}

A new SEM-EDS protocol was developed and shown to be a useful tool for determining size distributions and compositions of suspended particles. The method was compared to the PSD and chemical composition of the Arizona Test Dust standard. There was reasonable agreement between SEM results compared to other particlesizing methods for field samples collected in the coastal Long Island Sound and remote South Pacific, although many discrepancies were apparent that remain unresolved. An automated analysis with the use of scripts and MATLAB's Image Analysis Toolbox allowed for minimal user bias and a quick image processing and analysis time. Sample preparation has been optimized based on the linear relationship between beam attenuation and total suspended material to allow for greater accuracy in determining particle size distributions.

Acknowledgments. MST gratefully acknowledges support for this work from the U.S. Office of Naval Research Environmental Optics RaDyO DRI program, NASA Biology and Biogeochemistry, and NASA Contract NNX09AV55G. Steve Ackleson is thanked for enabling participation on the Long Island Sound cruise. For the BIOSOPE cruise, Davy Merien, Dominique Tailliez, and Claudie Bournot are warmly thanked for their help in the field. Emmanuel Boss and two anonymous reviewers provided helpful comments on the manuscript. This is a contribution of the BIOSOPE project of the LEFECYBER program. The BIOSOPE project was funded by the Centre National de la Recherche Scientifique (CNRS), the Institut des Sciences de l'Univers (INSU), the Centre National d'Etudes Spatiales (CNES), the European Space Agency (ESA), The National Aeronautics and Space Administration (NASA), and the Natural Sciences and Engineering Research Council of Canada (NSERC).

\section{REFERENCES}

Alldredge, A. L., and C. Gotschalk, 1988: In situ settling behavior of marine snow. Limnol. Oceanogr., 33, 339-351.

Allen, T., 1997: Powder Sampling and Particle Size Measurement. Vol. 1, Particle Size Measurement, 5th ed. Chapman and Hall, $525 \mathrm{pp}$.

Aurin, D., H. M. Dierssen, M. S. Twardowski, and C. S. Roesler, 2010: Optical complexity in Long Island Sound and implications for coastal ocean color remote sensing. J. Geophys. Res., 115, C07011, doi:10.1029/2009JC005837.

Babin, M., A. Morel, V. Fournier-Sicre, F. Fell, and D. Stramski, 2003: Light scattering properties of marine particles in coastal and open ocean waters as related to particle mass concentration. Limnol. Oceanogr., 48, 843-859.

Bader, H., 1970: The hyperbolic distribution of particle sizes. J. Geophys. Res., 75, 2822-2830.

Bernard, P. C., R. E. Van Grieken, and D. Eisma, 1986: Classification of estuarine particles using automated electron microprobe analysis and multivariate techniques. Environ. Sci. Technol., 20, 467-473. 
Bishop, J. K. B., and P. E. Biscaye, 1982: Chemical characterization of individual particles from the nepheloid layer in the Atlantic Ocean. Earth Planet. Sci. Lett., 58, 265-275.

Boss, E., and Coauthors, 2009: Comparison of inherent optical properties as a surrogate for particulate matter concentration in coastal waters. Limnol. Oceanogr. Methods, 7, $803-$ 810 .

Buonassissi, C., and H. M. Dierssen, 2010: A regional comparison of particle size distributions and the power-law approximation in oceanic and estuarine surface waters. J. Geophys. Res., 115, C10028, doi:10.1029/2010JC006256.

Chisholm, S. W., 1992: Phytoplankton size. Primary Productivity and Biogeochemical Cycles in the Sea, P. G. Falkowski and A. D. Woodhead, Eds., Plenum Press, 213-237.

Claustre, H., A. Sciandra, and D. Vaulot, 2008: Introduction to the special section bio-optical and biogeochemical conditions in the South East Pacific in late 2004: The BIOSOPE program. Biogeochemistry, 5, 679-691.

Danilatos, G.D., 1988: Foundations of Environmental Scanning Electron Microscopy. Vol. 71, Advances in Electronics and Electron Physics, Academic Press, 377 pp.

De Boer, D. H., and G. Crosby, 1995: Evaluating the potential of SEM/EDS analysis for fingerprinting suspended sediment derived from two contrasting topsoils. Catena, 24, 243-258.

Doucet, F. J., L. Maguire, and J. R. Lead, 2004: Size fractionation of aquatic colloids and particles by cross-flow filtration: Analysis by scanning electron and atomic force microscopy. Anal. Chim. Acta, 522, 59-71.

— , J. R. Lead, L. Maguire, E. P. Achterberg, and G. E. Millward, 2005: Visualisation of natural aquatic colloids and particlesA comparison of conventional high vacuum and environmental scanning electron microscopy. J. Environ. Monit., 7, $115-121$.

Gardner, W. D., and Coauthors, 2001: Optics, particles, stratification and storms on the New England continental shelf. J. Geophys. Res., 106, 9473-9497.

Harris, J. E., 1977: Characterization of suspended matter in the Gulf of Mexico. II: Particle size distribution of suspended matter from deep water. Deep-Sea Res., 58, 3322-3327.

Hunt, J. R., 1980: Prediction of oceanic particle size distributions from coagulation and sedimentation mechanisms. Particulates in Water, M. C. Kavanaugh and J. O. Leckie, Eds., Advances in Chemistry Series, Vol. 189, American Chemical Society, 243-257.

Jackson, G. A., R. Maffione, D. K. Costello, A. L. Alldredge, B. E. Logan, and H. G. Dam, 1997: Particle size spectra between $1 \mu \mathrm{m}$ and $1 \mathrm{~cm}$ at Monterey Bay determined using multiple instruments. Deep-Sea Res., 44, 1739-1767.

Jonasz, M., 1987: Nonsphericity of suspended marine particles and its influence on light scattering. Limnol. Oceanogr., 32, 10591065

- , and G. Fournier, 2007: Light Scattering by Particles in Water: Theoretical and Experimental Foundations. Academic Press, 714 pp.

Junge, C., 1963: Air Chemistry and Radioactivity. Academic Press, $382 \mathrm{pp}$.

Kahn, H., E. S. Mano, and M. M. M. L. Tassinari, 2002: Image analysis coupled with SEM-EDS applied to the characterization of a partially weathered $\mathrm{An}-\mathrm{Pb}$ ore. J. Miner. Mater. Charact. Eng., 1, 1-9.

Kim, J. P., J. Lemmon, and A. K. Hunter, 1995: Size-distribution analysis of sub-micron colloidal particles in river water. Environ. Technol., 16, 861-868.
Kitchen, J. C., 1977: Particle size distributions and the vertical distribution of suspended matter in the upwelling region off Oregon. Ph.D. thesis, Oregon State University, 130 pp.

Kostadinov, T. S., D. A. Siegel, and S. Maritorena, 2009: Retrieval of the particle size distribution from satellite ocean color observations. J. Geophys. Res., 114, C09015, doi:10/1029/2009JC005303.

Kutchko, B. G., and A. G. Kim, 2006: Fly ash characterization by SEM-EDS. Fuel, 85 (17-18), 2537-2544.

Lambert, C. E., C. Jehanno, N. Silverberg, J. C. Brun-Cottan, and R. Chesselet, 1981: Log-normal distributions of suspended particles in the open ocean. J. Mar. Res., 39, 77-98.

Lavoie, D. M., 1992: Computerized oceanic particle characterization using heavy metal staining, SEM, EDXS and image analysis. Deep-Sea Res., 39, 1655-1668.

Longhurst, A. R., and Coauthors, 1992: Sub-micron particles in northwest Atlantic shelf waters. Deep-Sea Res., 39, 1-7.

Mobley, C. D., and Coauthors, 1993: Comparison of numerical models for computing underwater light fields. Appl. Opt., 32, 7484-7504.

Morel, A., and B. Gentili, 1993: Diffuse reflectance of oceanic waters: II Bidirectional aspects. Appl. Opt., 32, 6864-6879.

- K. J. Voss, and B. Gentili, 1995: Bidirectional reflectance of oceanic waters: A comparison of modeled and measured upward radiance fields. J. Geophys. Res., 100 (C7), 13 143-13 150.

Myers, R. H., 1990: Classical and Modern Regression with Applications. Duxbury Press, 488 pp.

Neter, J., W. Wasserman, and M. H. Kutner, 1989: Applied Linear Regression Models. McGraw-Hill/Irwin, 720 pp.

Peng, F., D. L. Johnson, and S. W. Effler, 2002: Suspensoids in New York City's drinking water reservoirs: Turbidity apportionment. J. Amer. Water Resour. Assoc., 38, 1453-1465.

- S. W. Effler, D. O'Donnell, M. G. Perkins, and A. Weidemann, 2007: Role of minerogenic particles in light scattering in lakes and a river in central New York. Appl. Opt., 46, 6577-6594.

Pfitsch, D. W., E. Malkiel, Y. Ronzhes, S. King, J. Sheng, and J. Katz, 2005: Development of a free-drifting submersible digital holographic imaging system. Oceans 2005, Proc. MTS/IEEE, Marine Technology Society and IEEE, Washington, DC, 690696, doi:10.1109/OCEANS.2005.1639833.

,-- M. Takagi, Y. Ronzhes, S. King, J. Sheng, and J. Katz, 2007: Analysis of in-situ microscopic organism behavior in data acquired using a free-drifting submersible holographic imaging system. Oceans 2007, Proc. MTS/IEEE, Marine Technology Society and IEEE, Vancouver, BC, Canada, 1-8, doi:10.1109/OCEANS.2007.4449197.

Pilskaln, C. H., C. Lehmann, J. B. Paduan, and M. W. Silver, 1998: Spatial and temporal dynamics in marine aggregate abundance, sinking rate and flux: Monterey Bay, central California. Deep-Sea Res., 45, 1803-1837.

Reynolds, R. A., D. Stramski, V. M. Wright, and S. B. Wozniak, 2010: Measurements and characterization of particle size distributions in coastal waters. J. Geophys. Res., 115, C08024, doi:10.1029/2009JC005930.

Santschi, P. H., E. Balnois, K. J. Wilkinson, J. Zhang, and J. Buffle, 1998: Fibrillar polysaccharides in marine macromolecular organic matter as imaged by atomic force microscopy and transmission electron microscopy. Limnol. Oceanogr., 45, 896-908.

Sezgin, M., and B. Sankur, 2004: Survey over image thresholding techniques and quantitative performance evaluation. J. Electron. Imaging, 13, 146-165.

Sheng, J., E. Malkiel, and J. Katz, 2006: Digital holographic microscope for measuring three-dimensional particle distributions and motions. Appl. Opt., 45, 3893-3901. 
,,,--- J. Adolf, R. Belas, and A. Place, 2007: Digital holographic microscopy reveals prey-induced changes in swimming behavior of predatory dinoflagellates. Proc. Natl. Acad. Sci. USA, 104, 17 512-17 517.

$\longrightarrow,-$, and ——, 2008: Using digital holographic microscopy for simultaneous measurements of 3-dimensional near wall velocity and wall shear stress in a turbulent boundary layer. Exp. Fluids, 45, 1023-1035.

Sosik, H. M., and R. J. Olson, 2007: Automated taxonomic classification of phytoplankton sampled with imaging-in-flow cytometry. Limnol. Oceanogr. Methods, 5, 204-216.

Sournia, A., 1978: Phytoplankton Manual: Monographs on Oceanographic Methodology 6. Unesco, 337 pp.

Stramski, D., and D. A. Kiefer, 1991: Light scattering by microorganisms in the open ocean. Prog. Oceanogr., 28, 343-383.

- E. Boss, D. Bogucki, and K. Voss, 2004: The role of seawater constituents in light backscattering in the ocean. Prog. Oceanogr., 61, 27-56.

Syvitski, J. P. M., 1991: Principles, Methods, and Application of Particle Size Analysis. Cambridge University Press, $388 \mathrm{pp}$.

Twardowski, M. S., J. M. Sullivan, P. L. Donaghay, and J. R. V. Zaneveld, 1999: Microscale quantification of the absorption by dissolved and particulate material in coastal water with an ac9. J. Atmos. Oceanic Technol., 16, 691-707.

- E. Boss, J. B. Macdonald, W. S. Pegau, A. H. Barnard, and J. R. V. Zaneveld, 2001: A model for estimating bulk refractive index from the optical backscattering ratio and the implications for understanding particle composition in case I and case II waters. J. Geophys. Res., 106 (C7), 14129 14142 .
— M. Lewis, A. Barnard, and J. R. V. Zaneveld, 2005: Water instrumentation and platforms for ocean color remote sensing applications. Remote Sensing of Coastal Aquatic Waters, R. Miller and C. Del-Castillo, Eds., Springer-Kluwer, 69100.

- H. Claustre, S. A. Freeman, D. Stramski, and Y. Huot, 2007: Optical backscattering properties of the "clearest" natural waters. Biogeoscience, 4, 1041-1058.

_- and Coauthors, 2012: The optical volume scattering function in a surf zone inverted to derive sediment and bubble particle subpopulations. J. Geophys. Res., 117, C00H17, doi:10.1029/ 2011JC007347.

Vaillancourt, R. D., and W. M. Balch, 2000: Size distribution of marine submicron particles determined by flow field-flow fractionation. Limnol. Oceanogr., 45, 485-492.

Wells, M. L., and E. D. Goldberg, 1992: Marine submicron particles. Mar. Chem., 40, 5-18.

— Atlantic and Southern Oceans. Limnol. Oceanogr., 39, 286302.

Wilkinson, K. J., E. Balnois, G. G. Leppard, and J. Buffle, 1999: Characteristic features of the major components of freshwater colloidal organic matter revealed by transmission electron and atomic force microscopy. Colloids Surf., 155, 287-310.

Zaneveld, J. R. V., M. S. Twardowski, M. Lewis, and A. Barnard, 2005: Radiative transfer and remote sensing. Remote Sensing of Coastal Aquatic Waters, R. Miller and C. Del-Castillo, Eds., Springer-Kluwer, 1-20.

Zhang, X., M. S. Twardowski, and M. Lewis, 2011: Retrieving composition and sizes of oceanic particle subpopulations from the volume scattering function. Appl. Opt., 50, 1240-1259. 